\title{
First XMM-Newton observations of a Cataclysmic Variable II: The X-ray spectrum of OY Car ${ }^{\star}$
}

\author{
G. Ramsay ${ }^{1}$, F. Córdova ${ }^{2}$, J. Cottam ${ }^{3}$, K. Mason ${ }^{1}$, R. Much ${ }^{4}$, J. Osborne ${ }^{5}$, D. Pandel ${ }^{2}$, \\ T. Poole ${ }^{1}$, and P. Wheatley ${ }^{5}$
}

1 Mullard Space Science Lab., University College London, Holmbury St. Mary, Dorking, Surrey, RH5 6NT, UK

2 Department of Physics, University of California, Santa Barbara, CA 93106, USA

3 Columbia Astrophysics Laboratory, Columbia University, 538 West 120th Street, New York, NY 10027, USA

4 Astrophysics Division, ESTEC, 2200, AG Noordwijk, The Netherlands

5 Department of Physics \& Astronomy, University of Leicester, University Road, Leicester, LE1 7RH, UK

Received 27 September 2000 / Accepted 20 October 2000

\begin{abstract}
We present XMM-Newton X-ray spectra of the disc accreting cataclysmic variable OY Car, which were obtained during the performance verification phase of the mission. These data were taken 4 days after a short outburst. In the EPIC spectra we find strong iron $\mathrm{K} \alpha$ emission with weaker iron $\mathrm{K} \beta$ emission together with silicon and sulphur lines. The spectra are best fitted with a three temperature plasma model with a partial covering absorber. Multiple temperature emission is confirmed by the emission lines seen in the RGS spectrum and the $\mathrm{H} / \mathrm{He}$ like intensity ratio for iron and sulphur which imply temperatures of $\sim 7 \mathrm{keV}$ and $\sim 3 \mathrm{keV}$ respectively.
\end{abstract}

Key words. accretion, accretion discs - binaries: eclipsing - stars: individual: OY Car - novae, cataclysmic variables - X-rays: stars

\section{Introduction}

Cataclysmic variables $(\mathrm{CVs})$ are close binary systems in which the secondary (usually a dwarf main sequence star) fills its Roche lobe and transfers material onto the white dwarf primary. In non-magnetic systems, this material forms an accretion disc around the primary. Some of these systems show "dwarf nova" outbursts on the timescale of weeks to months when the system brightens by several magnitudes lasting a few days to months. During an outburst, the material close to the white dwarf is optically thick, while in quiescence the gas is optically thin (Pringle \& Savonije 1979; Narayan \& Popham 1993; Popham \& Narayan 1995).

X-ray observations of dwarf novae are hampered by their relatively low flux levels. With observations using large effective areas, such as XMM-Newton (Jansen et al. 2001), we can obtain high signal to noise X-ray spectra of dwarf novae for the first time. In this paper we present and analyse X-ray spectra of the dwarf nova OY Car. In a companion paper (Ramsay et al. 2001), we present and analyse the light curves of OY Car.

Send offprint requests to: G. Ramsay,

e-mail: gtbr@mssl.ucl.ac.uk

* Based on observations obtained with XMM-Newton, an ESA science mission with instruments and contributions directly funded by ESA Member States and the USA (NASA).

\section{Observations}

Ramsay et al. (2001) describe the XMM-Newton observations. Briefly, OY Car was observed twice using XMMNewton. The first observation (29-30 June 2000) was longer ( $\sim 50 \mathrm{ksec}$ in duration for the EPIC detectors) and had a higher count rate than the second observation (7 August 2000). This paper concentrates on the first observation which was made 4 days after a short outburst of OY Car.

The EPIC exposures were taken in full window mode using the medium filter. The particle background in both the EPIC detectors $(0.1-12 \mathrm{keV})$ (Turner et al. 2001) and the RGS (0.3-2.1 keV) (den Herder et al. 2001) increased significantly towards the end of the observation: the high background data were therefore removed from the analysis. Before extracting spectra of OY Car, the data were processed using the XMM-Newton Science Analysis System released on 12 July 2000.

\section{The RGS spectrum}

Although the mean background subtracted count rate was low $\left(\sim 0.05 \mathrm{ct} \mathrm{s}^{-1}\right)$ we were able to extract the RGS spectrum using data from both RGS1 and RGS2 (Fig. 1). Prominent emission lines are seen at $0.654 \mathrm{keV}$ from O VIII Ly $\alpha$ and at $1.473 \mathrm{keV}$ from Mg XII Ly $\alpha$. Fainter 


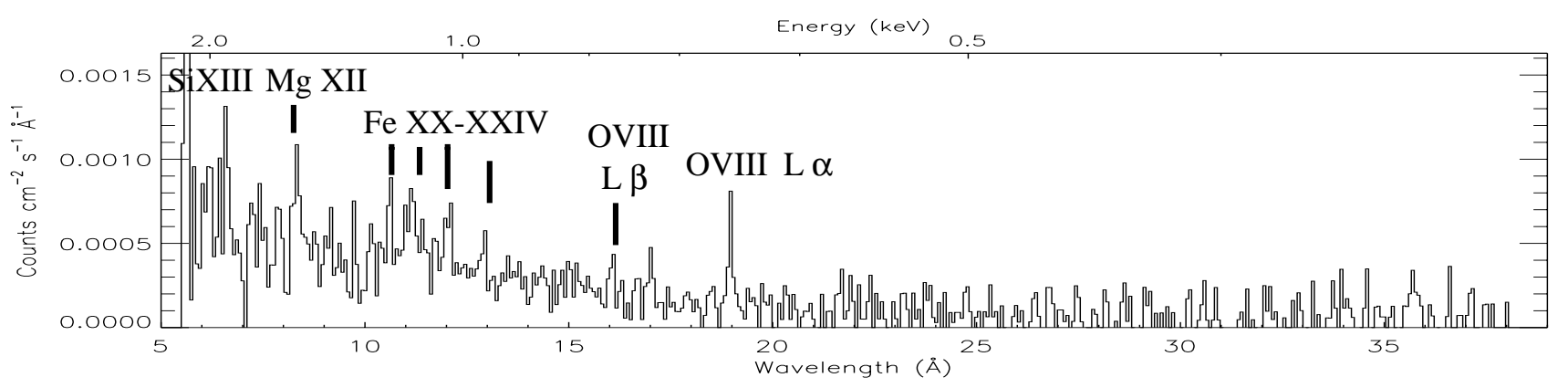

Fig. 1. The background subtracted RGS spectrum taken on 29 June 2000. The exposure was 55 ksec and includes both RGS1 and RGS2 data

lines are seen from O VIII Ly $\beta$ at $0.775 \mathrm{keV}$ and L-shell transitions of Fe XXIV to Fe XX between $1.16 \mathrm{keV}$ and $0.96 \mathrm{keV}$. There is a possible detection of Si XIII He-like series around $1.854 \mathrm{keV}$.

Given the statistical quality of the RGS data we are unable to perform detailed spectral analysis. We can, however, use the iron line detections to roughly constrain the temperature of the plasma assuming that it is in collisional equilibrium. The detection of $\mathrm{Fe} \mathrm{XX}$ lines requires a plasma temperature between 0.5 and $1.2 \mathrm{keV}$. However, the lack of emission from lower charge-states suggests that the temperature is actually higher than $\sim 0.8 \mathrm{keV}$. The simultaneous detection of Fe XXIV lines requires an additional temperature component, since these lines become prominent at temperatures larger than $\sim 1.3 \mathrm{keV}$. The lack of observable emission from the O VII He-like series but the detection of strong O VIII lines, puts a lowerlimit of $\sim 0.4 \mathrm{keV}$ on the oxygen emitting regions. The RGS spectrum suggests emission from a distribution of temperatures.

\section{The EPIC spectra}

\subsection{General features}

Spectra were extracted from all 3 EPIC cameras using apertures $\sim 30^{\prime \prime}$ in radius centered on OY Car, chosen so that the aperture did not cover more than one CCD. This encompasses $\sim 90$ percent of the integrated PSF (Aschenbach et al. 2000). Background spectra were extracted from the same CCD on which the source was detected, scaled and subtracted from the source spectra.

Since the response of the detectors is not well calibrated at present below $\sim 0.3 \mathrm{keV}$, energies below this were ignored in the following analysis. In our fits we used the response file mos1_medium_all_v3.14_15_tel2.rsp for the MOS detectors and epn_fs_Y9_medium.rmf for the PN detector. Since this PN response assumes only single pixel events, we extracted only these events to make our PN spectrum.

We show in Fig. 2 the integrated EPIC PN spectrum. Strong iron $\mathrm{K} \alpha$ emission lines are seen at $\sim 6.70$ and $6.94 \mathrm{keV}$, weaker iron $\mathrm{K} \beta$ line at $7.90 \mathrm{keV}$ and emission from Fe-L lines around $1.1 \mathrm{keV}$. Lines are also seen from Si and S. Similar lines are found in the spectra of the disc accreting CV SS Cyg (Done \& Osborne 1997) and the weakly magnetic CV EX Hya (Ishida et al. 1994).

\subsection{Spectral fitting}

The X-ray satellite $A S C A(0.6-10 \mathrm{keV})$ combined good spectral resolution together with reasonably large effective area. Around 15 dwarf novae were observed using ASCA. Amongst the brightest was SS Cyg. Done \& Osborne (1997) showed that in outburst a multi-temperature model was needed, while in quiescence a single-temperature model gave good fits to the data. We therefore fitted the integrated spectra from the three EPIC cameras simultaneously using models of increasing complexity.

A single temperature thermal plasma model with a model for absorption by neutral gas (the MEKAL and WABS models in XSPEC) gave a fit with $\chi_{\nu}^{2}=2.23$ (890 d.o.f.) and a best fit temperature of $k T=6.1 \mathrm{keV}$. Adding a second thermal plasma model yielded a significantly better fit: $\chi_{\nu}^{2}=2.06$ ( 888 d.o.f. $)(k T=3.3,8.0 \mathrm{keV})$. The normalisation of the various emission components was allowed to vary between the three EPIC spectra although their relative normalisations were held the same in each spectrum. Adding a third plasma model gave $\chi_{\nu}^{2}=1.87$ (886 d.o.f.) $(k T=0.8,3.3,7.7 \mathrm{keV})$. Using an F-test this model is better than the previous with a significance greater than 99.99 percent. The addition of yet another thermal plasma component did not give a significantly better fit nor did the addition of a blackbody component.

We note the lack of line emission at $6.4 \mathrm{keV}$. A line at $6.4 \mathrm{keV}$ has been seen in some dwarf novae (e.g. SS Cyg observed using ASCA: Done \& Osborne 1997) and is due to fluorescence either from the surface of the white dwarf or surrounding local material. By adding a Gaussian (fixed at $6.4 \mathrm{keV}$ ) to the model described above, we derive an upper limit on the equivalent width of $24 \mathrm{eV}$ for a line of width $100 \mathrm{eV}$ and $29 \mathrm{eV}$ for a line of width $50 \mathrm{eV}$. The fact that we did not detect a fluorescent line is possibly due to the high inclination of the system which may prevent us observing any reflection from the accretion disc.

For the sake of clarity, we show in Fig. 2 only the EPIC $\mathrm{PN}$ spectrum in full, together with the three temperature 


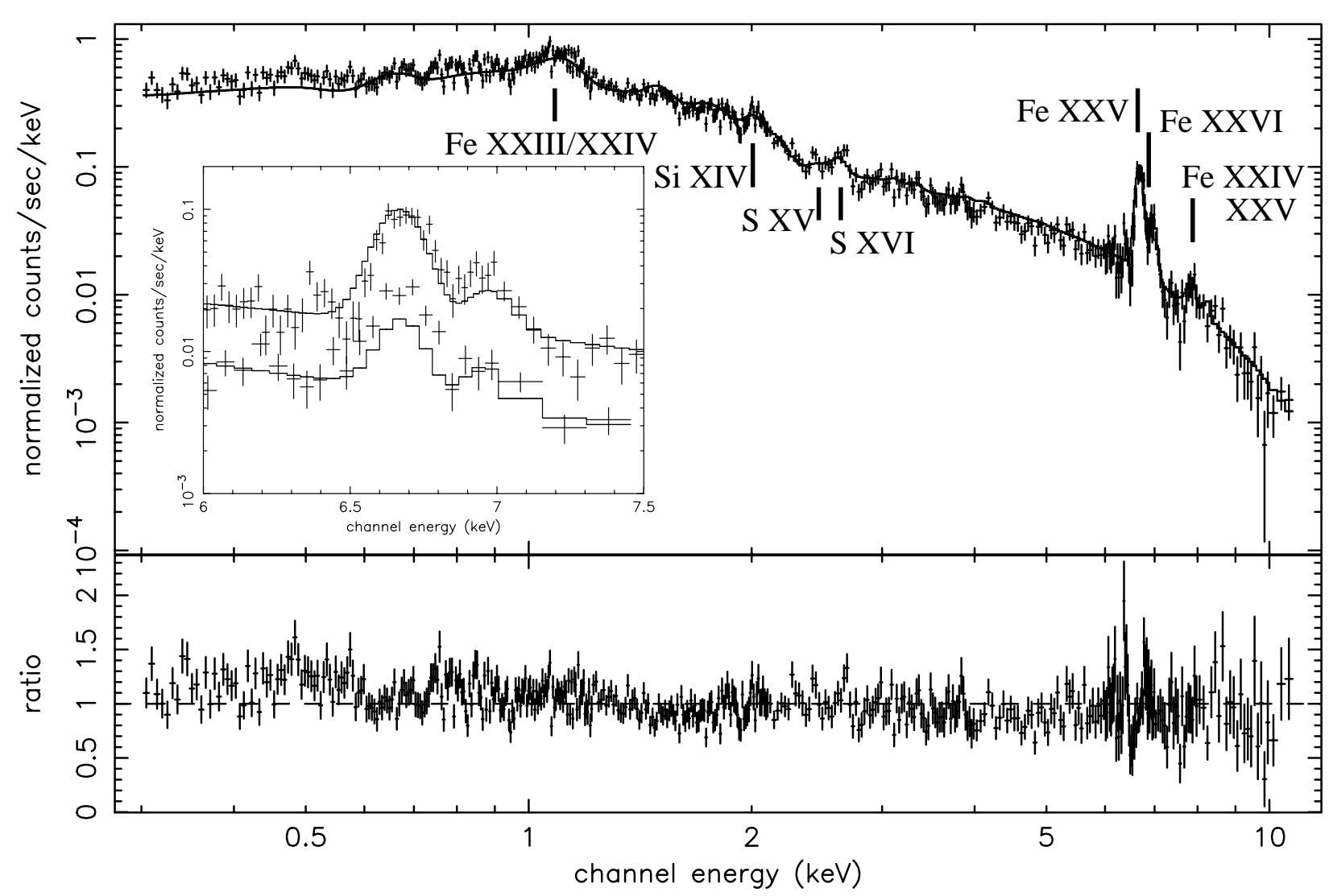

Fig. 2. Top panel: the integrated EPIC PN spectrum together with the best fit model using a neutral absorber and a three temperature thermal plasma model. Bottom panel: a plot of the data/model ratio. The inset shows the EPIC PN and MOS1 spectra with the best model fit covering $6.0-7.5 \mathrm{keV}$

Table 1. The best fit parameters for a simultaneous fit to all 3 integrated EPIC spectra using a 3 temperature MEKAL thermal plasma model

\begin{tabular}{lr}
\hline$N_{\mathrm{H}}\left(\mathrm{cm}^{-2}\right)$ & $4.8_{-0.4}^{+0.5} 10^{21}$ \\
Temperature (keV) & $7.7_{-0.4}^{+0.5}, 3.3_{-0.3}^{+0.2}$, \\
& $0.76_{-0.02}^{+0.04}$ \\
& $1.31_{-0.08}^{+0.06}$ \\
Metal abundance (solar) & $4.0 \pm 0.610^{-12}$ \\
Observed flux 0.2-10 keV & \\
$\left(\right.$ ergs s$\left.^{-1} \mathrm{~cm}^{-2}\right)$ & $5.0 \pm 1.010^{-12}$ \\
\hline Bolometric flux $\left(\mathrm{ergs} \mathrm{s}^{-1} \mathrm{~cm}^{-2}\right)$ & 5 \\
\hline
\end{tabular}

thermal plasma model and the residuals. We also show in Fig. 2 the EPIC PN and MOS1 spectra around $6.7 \mathrm{keV}$. Table 1 lists the best fit parameters. The need for a multitemperature model is consistent with the RGS spectrum which showed emission from plasma with more than one temperature.

In their observations of SS Cyg in quiescence Done \& Osborne (1997) found evidence that the absorption component is more complex than a simple neutral absorber. We therefore investigated if our X-ray spectra could be better modelled using a complex absorber. (In this study we only consider the EPIC PN data because of software problems in fitting all three EPIC spectra with complex absorbers.) When we fitted the EPIC PN spectrum alone we found that a two temperature MEKAL model with neutral absorption gave as good fits as a three temperature MEKAL model $\left(\chi_{\nu}^{2}=1.71,476\right.$ d.o.f $)$ : a third temperature component is not necessary. Using a warm absorber (of the sort described by Cropper et al. 1998) vary and fixing the column density of the neutral absorber to be $N_{\mathrm{H}}=310^{19} \mathrm{~cm}^{-2}$ (Mauche \& Raymond 2000) we obtain $\chi_{\nu}^{2}=1.61$ (475 d.o.f) with temperatures $k T=1.2$ and $6.0 \mathrm{keV}$ and a warm absorber of temperature $T \sim 510^{5} \mathrm{~K}$ and column density $N_{\mathrm{H}}=1.310^{21} \mathrm{~cm}^{-2}$. The implied value for the ionisation parameter is $\xi=0.058,\left(=L / n r^{2}\right.$ where $L$ is the luminosity, $n$ the density and $r$ the distance of the absorber from the irradiating source). The addition of the warm absorber produces significant absorption edges near $\sim 0.6 \mathrm{keV}$. The signal to noise of the RGS spectra is too low to detect these edges. This warm absorber model is significantly (>99.99 per cent using the F-test) better than the model which assumed only neutral absorption. Adding a third MEKAL model gave a fit $\left(\chi_{\nu}^{2}=1.59,473\right.$ d.o.f $)$ which is better than a two temperature model at the 97.7 per cent level. We also used a partial covering model instead of a warm absorber. 


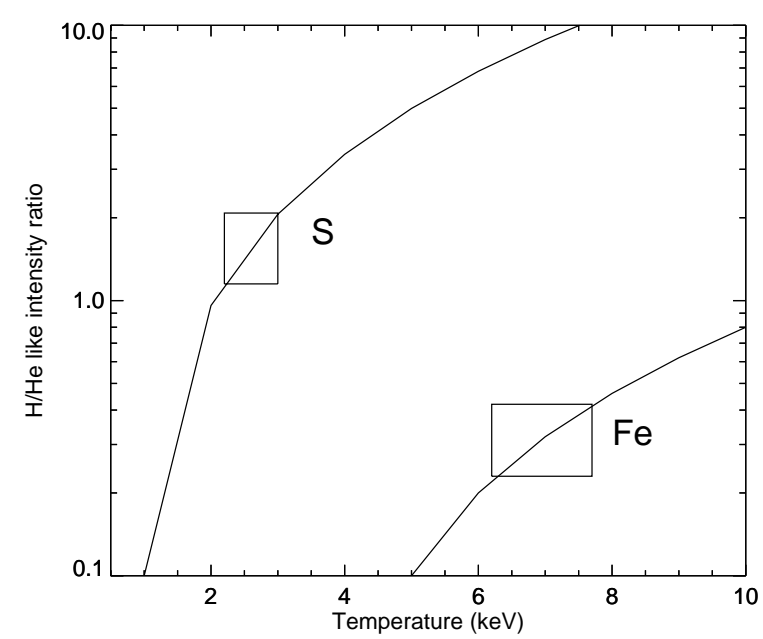

Fig. 3. The $\mathrm{H} / \mathrm{He}$ like intensity ratio for sulphur and iron $\mathrm{K} \alpha$ as a function of temperature. We plot the observed range determined using the EPIC PN spectrum

Using a two-temperature model gives a fit of $\chi_{\nu}^{2}=1.63$ (475 d.o.f). A three temperature model gave a significantly better fit $\left(\chi_{\nu}^{2}=1.54,473\right.$ d.o.f) with temperatures $(k T=0.7,2.5,7.2 \mathrm{keV})$ and a partial covering model of column $N_{\mathrm{H}}=6.310^{21} \mathrm{~cm}^{-2}$ with a covering fraction of 0.51 .

We conclude that there is evidence for complex absorption in the X-ray spectrum of OY Car. We caution, however, that we have used the integrated spectrum in our study: Ramsay et al. (2001) found evidence for a spectral variation in the light curves. It is left to a future paper to investigate this.

\subsection{Line studies}

We can also determine the temperature of the plasma by measuring the intensity ratio of the $\mathrm{H}$ and He like line emission. Using the EPIC PN spectrum we can determine this ratio for $\mathrm{Fe}$ and $\mathrm{S}$. We fitted a thermal bremsstrahlung emission component and added Gaussian components to fit the $\mathrm{He}$ and $\mathrm{H}$ like emission lines. For $\mathrm{Fe}$ at 6.70 and $6.95 \mathrm{keV}$ we find a $\mathrm{H} / \mathrm{He}$ like intensity ratio of $0.31_{-0.08}^{+0.11}$ and for $\mathrm{S}$ at 2.43 and $2.65 \mathrm{keV}$ we find a ratio of $1.15_{-0.06}^{+0.87}$. Using the MEKAL thermal plasma model in XSPEC we obtained the $\mathrm{H} / \mathrm{He}$ like intensity ratio verses ionisation temperature for the metal abundance derived in the fit (Table 1) and plot this in Fig. 3. We find these ratios give temperatures of $6-8 \mathrm{keV}$ for iron $\mathrm{K} \alpha$ and $2-3 \mathrm{keV}$ for sulphur. Thus, as in our fits using multi-temperature MEKAL models, we need a range of temperatures to model the data. Indeed the temperatures derived from the line fits are consistent with the temperatures of the hotter two thermal plasma components.

\subsection{The luminosity}

The flux values shown in Table 1 are the mean values determined from each EPIC spectrum. For a distance of $82 \pm 12$ pc (Wood et al. 1989) we determine the bolometric X-ray luminosity to be $4.0 \pm 0.810^{30} \mathrm{ergs} \mathrm{s}^{-1}$. This luminosity is similar to that reported for other nonmagnetic CVs in quiescence: Pratt et al. (1999) found $L_{\mathrm{X} \text {,bol }} \sim 10^{30} \mathrm{ergs}^{-1}$ for OY Car using ROSAT data. The luminosity in the ROSAT energy band $(0.1-2 \mathrm{keV})$ is $1.910^{30} \mathrm{ergs} \mathrm{s}^{-1}$. We can determine the accretion rate, $\dot{M}$, from $L_{\text {acc }}=G M_{1} \dot{M} / R_{1}$, where $L_{\text {acc }}$ is the accretion luminosity and $L_{\mathrm{bl}}=L_{\mathrm{acc}} / 8$ (Popham \& Narayan 1995), where $L_{\mathrm{bl}}$ is the boundary layer luminosity. For a white dwarf mass of $1.0 M_{\odot}$ (Ramsay et al. 2001) and assuming the X-ray flux originates mainly from the boundary layer, we find a mass accretion rate during quiescence of $1.310^{14} \mathrm{~g} \mathrm{~s}^{-1}$ or $1.910^{-12} M_{\odot} \mathrm{yr}^{-1}$. The true luminosity and accretion rate maybe significantly higher since van Teeseling et al. (1996) showed that the observed flux is anti-correlated with inclination: for high inclination systems most of the flux is absorbed by the accretion disc.

\section{Summary}

We have examined the X-ray spectra of OY Car obtained using XMM-Newton. These spectra have a much higher signal to noise and spectral resolution than previous X-ray spectra of dwarf nova. We find strong emission lines of various ionisation species. In fitting the EPIC spectra we require a multi-temperature plasma. This is confirmed by the line species seen in the RGS spectrum and also from the $\mathrm{H} / \mathrm{He}-\mathrm{like}$ intensity ratios of iron and sulphur. Adding a more complex absorber such as a warm absorber or a partial covering model to a neutral absorber significantly improves the fit to the data.

\section{References}

Aschenbach, B., Briel, U., Haberl, F., et al. 2000, SPIE, 4012, Paper 86 [astro-ph/0007256]

Cropper, M., Ramsay, G., \& Wu, K. 1998, MNRAS, 293, 222

den Herder, J. W., Brinkman, A. C., Kahn, S. M., et al. 2001, A\&A, 365, L7

Done, C., \& Osborne, J. P. 1997, MNRAS, 288, 649

Ishida, M., Mukai, K., \& Osborne, J. P. 1994, PASJ, 46, L81

Jansen, F., Lumb, D., Altieri, B., et al. 2001, A\&A, 365, L1

Mauche, C., \& Raymond, J. C. 2000, ApJ, 541, 924

Narayan, R., \& Popham, R. 1993, Nat, 362, 820

Popham, R., \& Narayan, R. 1995, ApJ, 442, 337

Pratt, G. W., Hassall, B. J. M., Naylor, T., Wood, J. H., \& Patterson, J. 1999, MNRAS, 309, 847

Pringle, J. E., \& Savonije, G. J. 1979, MNRAS, 187, 777

Ramsay, G., Poole, T., Mason, K., et al. 2001, A\&A, 365, L288

Turner, M. J. L., Abbey, A., Arnaud, M., et al. 2001, A\&A, 365, L27

Wood, J. H., Horne, K., Berriman, G., \& Wade, R. A. 1989, ApJ, 341, 974

van Teeseling, A., Beuermann, K., \& Verbunt, F. 1996, A\&A, 315,467 